\title{
Classification of Swiss cheese starter and adjunct cultures using Fourier transform infrared microspectroscopy
}

\author{
V. Prabhakar, N. Kocaoglu-Vurma, J. Harper, and L. Rodriguez-Saona ${ }^{1}$ \\ Department of Food Science and Technology, The Ohio State University, 110 Parker Food Science and Technology Building, 2015 Fyffe Rd., \\ Columbus 43210
}

\begin{abstract}
The acceptability of Swiss cheese largely depends on the flavor profile, and strain variations of cheese cultures will affect the final quality. Conventional biochemical methods to identify the cultures at the strain level are time-consuming and expensive, and require skilled labor. Our objective was to develop rapid classification methods of starter cultures at the strain level by using a combination of hydrophobic grid membrane filters and Fourier transform infrared (FTIR) spectroscopy. Forty-four pulsed-field gel electrophoresis-verified strains of starter and nonstarter cultures including Streptococcus thermophilus, Propionibacterium freudenreichii, and Lactobacillus spp. were analyzed. The strains were grown on their respective agar media, transferred to broth media, and incubated. Then, cultures were centrifuged and the pellets were resuspended in saline solution $(10 \mu \mathrm{L})$. Aliquots $(2 \mu \mathrm{L})$ of the suspended bacterial solution were placed onto a grid of the hydrophobic grid membrane filters, having 6 grids per each strain analyzed. The dried filters were read by FTIR microspectroscopy fitted with an attenuated total reflectance probe. Collected spectra were statistically analyzed by a soft independent modeling of class analogy (SIMCA) for pattern recognition. Classification models were developed for Streptococcus thermophilus, Propionibacterium freudenreichii, and Lactobacillus spp. strains. The models showed major discrimination in the spectral region from 1,200 to 900 $\mathrm{cm}^{-1}$ associated with signals from phosphate-containing compounds and various polysaccharides in the cell wall. The developed method allowed for rapid classification of several Swiss cheese starter and nonstarter cultures at the strain level. This information provides a detailed overview of microbiological status, which would enable corrective measures to be taken early in the cheese making process, limiting production of inferior quality cheese and minimizing defects. This method could
\end{abstract}

Received April 16, 2011.

Accepted May 9, 2011.

${ }^{1}$ Corresponding author: rodriguez-saona.1@osu.edu be an effective tool to identify and monitor activity of cheese and other dairy starter cultures.

Key words: Fourier transform infrared spectroscopy, Swiss cheese, microbiological identification, starter and nonstarter culture

\section{INTRODUCTION}

Swiss cheese is a hard cheese prepared from pasteurized milk and has holes, or eyes, that develop throughout the cheese due to microbiological activity (USDA, 2001). Although the Swiss cheese-making process differs among various countries, the basic steps involved in Swiss cheese making are milk coagulation, removal of whey, acid production, salt addition, and ripening. Initial ripening is carried out in warm room for the eye formation to take place by the action of propionibacteria, and the cheese is moved to cold room to finish the ripening process (Beresford et al., 2001). The quality of Swiss cheese essentially depends on fermentation by lactic acid and propionic acid bacteria, and proteolysis (Steffen et al., 1987). Thermophilic lactic acid bacteria (LAB), such as Streptococcus thermophilus and Lactobacillus helveticus, and propionic acid bacteria, mostly Propionibacterium freudenreichii ssp. shermanii, are common starter cultures in Swiss cheese making (Ji et al., 2004).

In addition to the starter cultures, Swiss cheese microflora also comprises nonstarter lactic acid bacteria (Fox et al., 2000) that usually consist of facultatively heterofermentative lactobacilli such as Lactobacillus casei, Lactobacillus delbrueckii, Lactobacillus plantarum, or Lactobacillus rhamnosus (Weinrichter et al., 2004). Although nonstarter lactic acid bacteria are generally indigenous to the raw milk or cheese-manufacturing facility, intentional addition of selected strains as adjunct cultures has been implemented because of their ability to accelerate proteolysis for cheese flavor development (Kieronczyk et al., 2003). It has been reported that facultatively heterofermentative lactobacilli adjunct starters may influence the characteristics of Swiss cheeses with respect to various properties (Weinrichter et al., 2004) and that cheese manufactured with adjunct lactobacilli showed better flavor intensity than cheeses made 
from pasteurized or microfiltered milk, suggesting that indigenous nonstarter lactic acid bacteria play a role in the development of cheese flavor (Lynch et al., 1997).

Overall, the complex microflora has a major effect on cheese ripening and hence the characteristic features of Swiss cheese. Lortala and Chapot-Chartier (2005) reported that lysis of $\mathrm{LAB}$ is an essential parameter to control and accelerate cheese ripening. However, knowledge about genetic similarities and differences are needed for rational culture selection because genetic variations are correlated with cheese making and cheese quality parameters (Jenkins et al., 2002). Selection of suitable strains would enable the cheese maker to control or modify flavor development (Beresford et al., 2001). Evaluating the presence, numbers, and types of starter bacteria is of utmost importance for the dairy industry because it allows for identification of the bacterial flora of starter cultures, detection of the total numbers in dairy samples, and detection of particular strains or biotypes present in dairy products.

Conventional methods used to characterize the cheese starter cultures are summarized in many publications (Holzapfel et al., 2001; Tannock, 2001; Jenkins et al., 2002). These methods include SDS-PAGE of whole-cell proteins, DNA-DNA hybridization, and PCR. Strainlevel differentiation typically uses PCR-based methods or methods based on restriction enzyme analysis (Kahala et al., 2008). Several studies report that pulsed-field gel electrophoresis (PFGE) is a highly reproducible method for characterization of starter cultures at the strain level (Tanskanen et al., 1990; Tilsala-Timisjarvi and Alatossava, 1997; Mannu and Paba, 2002; Lick, 2003; Ward et al., 2004). However, these biochemical tests and molecular typing techniques are expensive and time-consuming, and require extensive skill, especially for the identification of bacteria at the subspecies or strain level (Oust et al., 2004; Dziuba et al., 2007).

Fourier transform infrared (FTIR) spectroscopy is an attractive technology for the rapid, inexpensive, sensitive, and high-throughput analysis of microorganisms. The FTIR method allows for the chemically based discrimination of intact microbial cells and produces complex biochemical fingerprints that are distinct and reproducible for different bacteria. Bacteria have shown highly specific mid-infrared spectral patterns that may be unique for individual strains (Helm et al., 1991; Naumann et al., 1991). Fourier transform infrared spectroscopy combined with chemometrics has been used for the accurate classification of microorganisms (Wenning et al., 2002; Mossoba et al., 2003; Ngo-Thi et al., 2003). Moreover, FTIR microspectroscopy combined with hydrophobic grid membrane filters (HGMF) was successfully used for rapid characterization of Salmonella serovars (Männig et al., 2008). Hydrophobic grid membrane filters have a special printed grid pattern that separates bacterial colonies from one another, thus preventing lateral growth, spreading, and confluence.

The objective of this study was to classify Swiss cheese starter and adjunct cultures at the strain level using a combination of HGMF, FTIR microspectroscopy, and multivariate analysis.

\section{MATERIALS AND METHODS}

\section{Bacterial Cultures}

Thirteen strains of Streptococcus thermophilus, 21 strains of Lactobacillus spp., and 10 strains of Propionibacterium freudenreichii were used for this study (Table 1). Detailed information of the bacterial cultures can be

Table 1. List of Swiss cheese starter and nonstarter culture strains

Strain

Streptococcus thermophilus S363

Strep. thermophilus $\mathrm{S} 804$

Strep. thermophilus A054

Strep. thermophilus S341

Strep. thermophilus S392

Strep. thermophilus 884

Strep. thermophilus $\mathrm{S} 869$

Strep. thermophilus S838

Strep. thermophilus S847

Strep. thermophilus S728

Strep. thermophilus $\mathrm{S} 765$

Strep. thermophilus S731

Strep. thermophilus S794

Lactobacillus casei $\mathrm{A} 2$

L. casei A26

L. casei $\mathrm{A} 3$

L. casei A34

Lactobacillus fermentum B4

L. fermentum B15

L. casei $\mathrm{B} 21$

Lactobacillus delbrueckii $\mathrm{C} 2$

Lactobacillus rhamnosus D56

L. delbrueckii E3

L. delbrueckii F1

L. delbrueckii $\mathrm{F} 44$

L. casei $\mathrm{G} 2$

L. rhamnosus $\mathrm{H} 1$

L. rhamnosus $\mathrm{H} 2$

Lactobacillus helveticus $\mathrm{S} 1$

L. helveticus $\mathrm{S} 2$

L. helveticus $\mathrm{S} 9$

L. helveticus S14

L. helveticus $\mathrm{S} 15$

L. helveticus S5

Propionibacterium freudenreichii $\mathrm{P} 891$

P. freudenreichii $\mathrm{P} 812$

P. freudenreichii $\mathrm{P} 728$

P. freudenreichii $\mathrm{P} 843$

P. freudenreichii $\mathrm{P} 835$

P. freudenreichii $\mathrm{P} 859$

P. freudenreichii P318

P. freudenreichii $\mathrm{P} 745 \mathrm{M} 1$

P. freudenreichii P745M2

P. freudenreichii ATCC 6207 
found in Tables 2, 3, and 4. The cultures were provided by the J. T. Parker Chair Swiss cheese culture collection. The cultures and company names were coded for confidentiality. The letter in the code refers to the strain genus $(\mathrm{L}=$ Lactobacillus, $\mathrm{P}=$ Propionibacterium, and $\mathrm{S}=$ Streptococcus), the first number of each code indicates the culture manufacturer, and the last 2 numbers are the strain designation. Cultures containing multiple strains were designated with an " $\mathrm{M}$ " following the numerical code. All the culture strains were previously identified by PFGE (Jenkins et al., 2002). Streptococcus thermophilus strains were grown in M17 agar containing $0.5 \%$ lactose and $0.15 \%$ lithium chloride for $2 \mathrm{~d}$ at $42^{\circ} \mathrm{C}$. Colonies were transferred to M17 broth containing $0.5 \%$ lactose and $0.15 \%$ lithium chloride and incubated at $42^{\circ} \mathrm{C}$ for $24 \mathrm{~h}$. Strains of Lactobacillus spp. were grown on Rogosa SL agar for $2 \mathrm{~d}$ at $37^{\circ} \mathrm{C}$ in an anaerobic chamber (Forma Scientific Inc., Marietta, $\mathrm{OH}$ ) purged with a $5 \%$ carbon dioxide, $10 \%$ hydrogen, $85 \%$ nitrogen gas mixture. Colonies were transferred to lactobacilli de Man, Rogosa, and Sharpe broth and incubated for $24 \mathrm{~h}$ at $37^{\circ} \mathrm{C}$ in an anaerobic chamber. Propionibacterium freudenreichii strains were grown anaerobically in sodium lactate agar for 5 to $7 \mathrm{~d}$ at $30^{\circ} \mathrm{C}$. Colonies were transferred to sodium lactate broth and incubated anaerobically for $2 \mathrm{~d}$ at $30^{\circ} \mathrm{C}$. Bacterial cultures were analyzed on 4 separate days.

\section{Sample Preparation}

Individual bacterial cultures grown in broth medium $(1 \mathrm{~mL})$ were centrifuged $\left(5,934 \times g ; 4^{\circ} \mathrm{C} ; 5 \mathrm{~min}\right)$ and washed with $0.85 \%$ saline solution; the resulting bacterial pellets were resuspended in $10 \mu \mathrm{L}$ of saline solution. An aliquot $(2 \mu \mathrm{L})$ of the suspension was applied onto 1 grid of HGMF (0.45- $\mu \mathrm{m}$ porous, Neogen Corp., Lansing, MI) placed on an Iso-Grid filtration unit (Iso-Grid, Neogen Corp.). Six grids were used for each bacterial strain and the HGMF containing the cultures were dried in a vacuum desiccator for 5 min before analysis.

\section{FTIR Microspectroscopy}

The HGMF containing bacterial cultures were analyzed using an FTIR microscope (UMA 600 series infrared microscope interfaced with a FTS Excalibur 3100GX FTIR spectrometer; Varian, Walnut Creek, CA) equipped with a mercury cadmium telluride detector in attenuated total reflectance mode. The microscope was equipped with a motorized $\mathrm{x}-\mathrm{y}$ stage, $4 \times$ and $16 \times$ objectives, and a slide-on attenuated total reflection germanium (Ge) objective (600 UMA, Varian, Palo Alto, CA).The instrument had a Permaglow (ceramic; Varian Inc., Palo Alto, CA) mid-infrared source and an extended-range potassium bromide beam splitter. The FTIR spectra were collected using Varian Resolutions Pro (v4.05, Varian Inc., Palo Alto, CA) in the mid-infrared region $\left(4,000\right.$ to $\left.700 \mathrm{~cm}^{-1}\right)$ at 8 $\mathrm{cm}^{-1}$ resolution. The spectrum of the Ge surface alone was measured as a background reference before sample analysis. To enhance the signal-to-noise ratio, the sample and background single beam Fourier-processed spectra consisted of 128 scans that were co-added and averaged. The absorbance spectra were determined by the ratio of the sample's single beam spectra to background. Six spectra per individual cheese culture strain were collected. The reproducibility of cultures grown on 3 to 5 different days was also examined, resulting in 18 to 30 spectra per strain to construct the training model.

\section{Multivariate Analysis}

Multivariate analysis of the collected spectral data was performed using the Pirouette software (Infometrix Inc., Bothel, WA). The FTIR spectra were mean-centered, transformed to their second derivative

Table 2. Interclass distances between 13 Streptococcus thermophilus strains

\begin{tabular}{|c|c|c|c|c|c|c|c|c|c|c|c|c|c|}
\hline Strain & S363 & S804 & $\mathrm{A} 054$ & S341 & S393 & S884 & S869 & S838 & S847 & S728 & S765 & S731 & S794 \\
\hline S804 & 6.2 & 0.0 & & & & & & & & & & & \\
\hline A054 & 3.9 & 5.9 & 0.0 & & & & & & & & & & \\
\hline S393 & 7.3 & 7.1 & 4.4 & 6.2 & 0.0 & & & & & & & & \\
\hline S884 & 10.5 & 5.6 & 10.4 & 7.2 & 10.4 & 0.0 & & & & & & & \\
\hline S869 & 7.5 & 6.7 & 7.3 & 9.2 & 7.7 & 9.1 & 0.0 & & & & & & \\
\hline S728 & 6.1 & 6.6 & 3.3 & 7.4 & 5.1 & 11.0 & 9.3 & 5.2 & 5.7 & 0.0 & & & \\
\hline S765 & 2.3 & 7.2 & 3.6 & 10.8 & 7.6 & 12.1 & 8.0 & 3.5 & 3.5 & 5.4 & 0.0 & & \\
\hline S731 & 2.4 & 6.5 & 3.5 & 9.7 & 6.8 & 10.3 & 6.3 & 3.6 & 3.2 & 5.4 & 1.4 & 0.0 & \\
\hline S794 & 4.4 & 6.0 & 3.2 & 8.9 & 6.8 & 10.3 & 6.5 & 4.6 & 4.5 & 6.7 & 5.2 & 4.0 & 0.0 \\
\hline
\end{tabular}


Table 3. Interclass distances between 21 Lactobacillus spp. strains ${ }^{1}$

\begin{tabular}{|c|c|c|c|c|c|c|c|c|c|c|c|c|c|c|c|c|c|c|c|c|c|}
\hline Strain & $\mathrm{A} 2$ & A 26 & A3 & A34 & B4 & B15 & B21 & C2 & D56 & E3 & $\mathrm{F} 1$ & F44 & G2 & H1 & H2 & S1 & S2 & S9 & S14 & S15 & S5 \\
\hline A26 & 4.4 & 0.0 & & & & & & & & & & & & & & & & & & & \\
\hline A3 & 6.3 & 1.2 & 0.0 & & & & & & & & & & & & & & & & & & \\
\hline B4 & 4.8 & 3.3 & 4.2 & 4.5 & 0.0 & & & & & & & & & & & & & & & & \\
\hline B15 & 13.7 & 9.9 & 13.4 & 12.6 & 8.4 & 0.0 & & & & & & & & & & & & & & & \\
\hline B21 & 2.8 & 3.4 & 5.1 & 2.4 & 3.5 & 13.4 & 0.0 & & & & & & & & & & & & & & \\
\hline E3 & 11.2 & 8.3 & 10.1 & 10.5 & 6.8 & 8.7 & 10.7 & 10.1 & 11.0 & 0.0 & & & & & & & & & & & \\
\hline F1 & 8.8 & 5.9 & 7.5 & 8.2 & 5.3 & 10.4 & 8.0 & 7.1 & 8.7 & 4.3 & 0.0 & & & & & & & & & & \\
\hline F44 & 6.5 & 5.1 & 6.9 & 6.1 & 5.8 & 13.6 & 6.1 & 4.8 & 6.3 & 10.9 & 8.1 & 0.0 & & & & & & & & & \\
\hline G2 & 4.9 & 3.2 & 4.9 & 4.4 & 4.8 & 14.9 & 3.6 & 5.9 & 4.3 & 11.6 & 8.9 & 5.6 & 0.0 & & & & & & & & \\
\hline H1 & 7.2 & 3.6 & 5.0 & 6.8 & 6.0 & 15.2 & 7.3 & 8.4 & 4.9 & 12.8 & 10.5 & 8.4 & 7.7 & 0.0 & & & & & & & \\
\hline H2 & 3.3 & 4.3 & 6.6 & 3.0 & 4.4 & 14.4 & 3.2 & 6.1 & 4.6 & 11.7 & 9.7 & 6.4 & 4.8 & 7.7 & 0.0 & & & & & & \\
\hline S1 & 6.7 & 6.7 & 8.8 & 6.3 & 5.1 & 12.0 & 6.6 & 4.9 & 7.8 & 10.8 & 9.1 & 7.4 & 8.0 & 10.3 & 6.4 & 0.0 & & & & & \\
\hline
\end{tabular}

${ }^{1}$ Strains A2, A26, A3, A34, B21, and G2 = Lactobacillus casei; strains B4 and B15 = Lactobacillus fermentum; strains C2, E3, F1, and F44 = Lactobacillus delbrueckii; strains D56, H1, and H2 = Lactobacillus rhamnosus; strains S1, S9, S14, S15, and S5 = Lactobacillus helveticus.

using a 5-point Savitzky-Golay polynomial filter, and normalized before analysis. Soft independent modeling of class analogy (SIMCA) was used to build classification models for the cheese cultures. The SIMCA analysis procedure consists of assigning a separate class for each sample and creating a principal component analysis model to explain the majority of variation in the data set and grouping samples based on similarities (De Maesschalck et al., 1999). The first 3 principal components are used to project the classes; however, more principal components may be used for the actual differentiation. Inter-class distances were calculated using between-class residuals, and variable importance was determined by comparing the average residual variance of each class to all classes and the residual variance of all classes to themselves. Variable importance, also known as discriminating power, was used to define the variables that have a predominant effect on sample classification (Dunn and Wold, 1995).

\section{RESULTS AND DISCUSSION}

Swiss cheese cultures were isolated using HGMF, and infrared microspectroscopy was used for direct fingerprinting of starter and nonstarter (adventitious bacterial flora) cultures involved in cheese production. The application of HGMF allowed for isolation of the bacterial culture in a hydrophobic square, limiting horizontal spread and overlap of microcolonies. Representative raw spectrums of Strep. thermophilus, L. delbrueckii, and P. freudenreichii are shown in Figure 1. Transformation of raw bacterial spectra to its second derivative using Savitzky-Golay second derivative algorithm (5-point gap size) allowed for reduced variability between rep-

Table 4. Interclass distances between 10 Propionibacterium freudenreichii strains

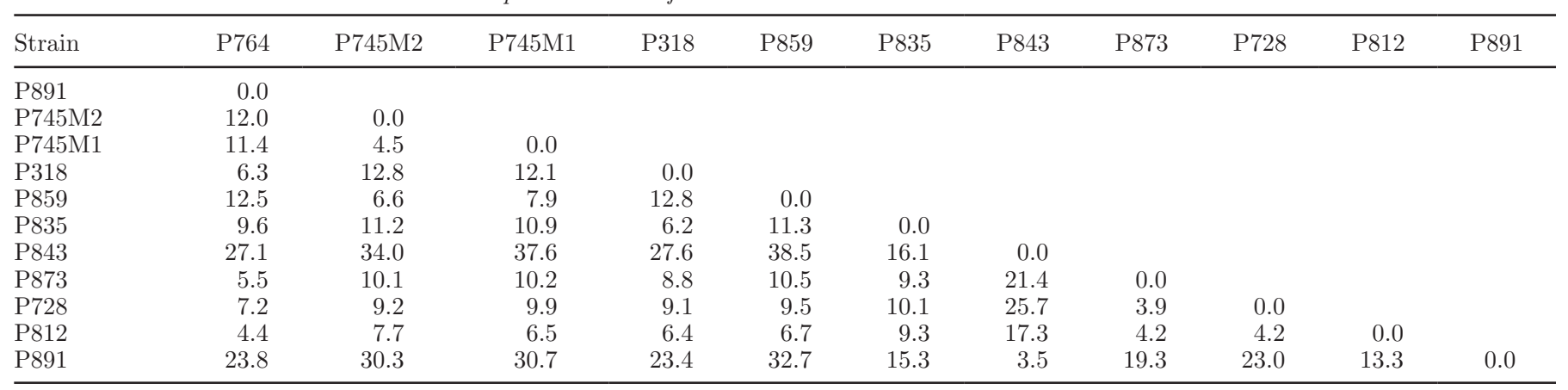




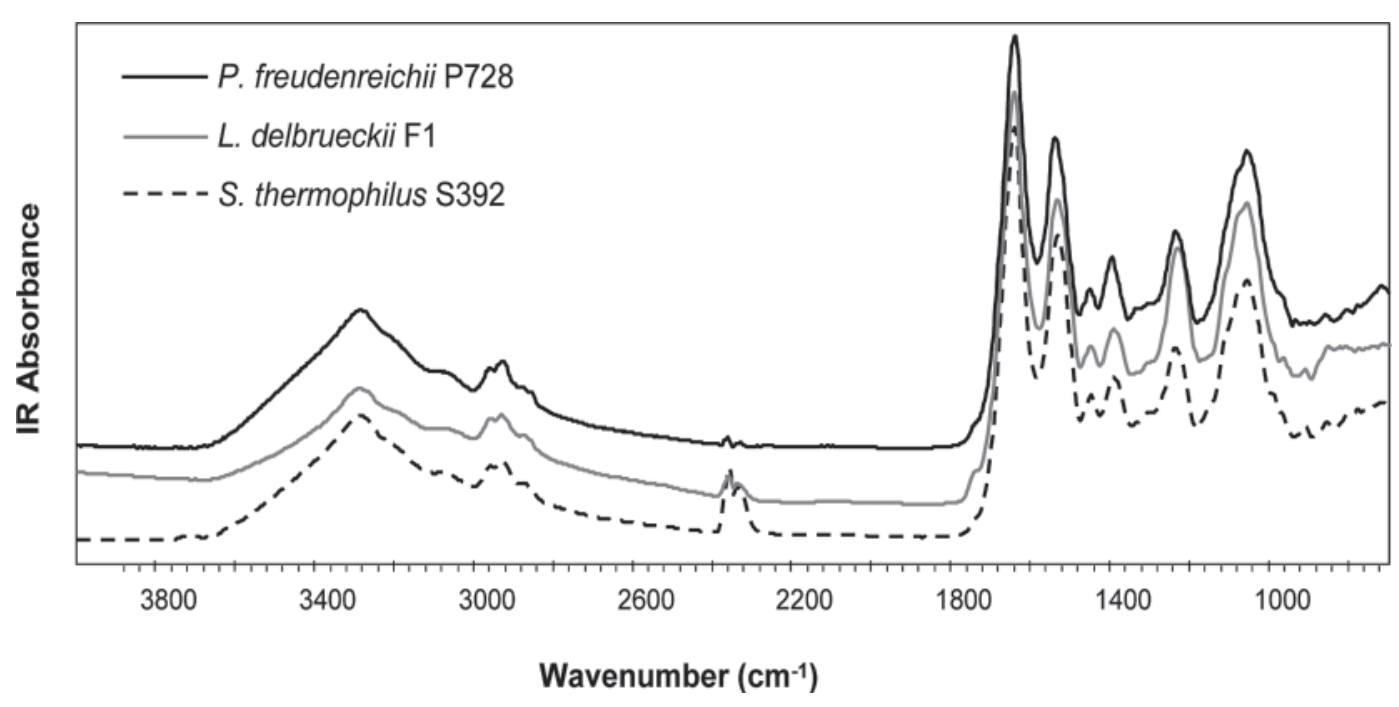

Figure 1. Typical Fourier transform infrared raw spectra of Streptococcus thermophilus, Lactobacillus delbrueckii, and Propionibacterium freudenreichii bacteria.

lications by removing baseline variation and resolving overlapping peaks (Kansiz et al., 2001).

Multivariate analysis of spectra resulted in classification models for differentiating between strains of Strep. thermophilus, Lactobacillus spp., and P. freudenreichii, the main starter and adjunct cultures used in making Swiss cheese (Ji et al., 2004). This protocol allowed for the collection of reproducible infrared absorption spectra directly from biomass of individual colonies isolated with HGMF, thus overcoming the labor-intensive and time-consuming biochemical and phenotypic characterization of bacteria of traditional methods for identification of cheese microorganisms such as PFGE and DNA fingerprinting. Species differentiation based on infrared spectroscopic data has been shown to be consistent with the analysis results obtained by the $16 \mathrm{~S}$ RNA sequencing, regarded as the gold standard (Kirschner et al., 2001).

The SIMCA analysis of FTIR spectral data from 13 Strep. thermophilus cultures showed good clustering at the strain level (Figure 2A) and good separation between classes, evidencing the potential of infrared spectroscopy and multivariate analysis to detect subtle differences for intraspecific differentiation of strains. The SIMCA model (Figure 2A) showed close grouping between the strains S363, A054, S838, S847, S765, S731, and S794, suggesting high similarities between these 7 strains that was confirmed by interclass distance (ICD) values (Table 2) close to 3 ; generally, ICD $>3$ are indicative of good discrimination (Kyalheim and Karstang, 1992). All other strains showed ICD values $>3$. Cluster analysis based on PFGE for Strep. thermophilus cultures used for Swiss cheese manufac- ture has shown 30 to $40 \%$ similarity among cultures (O'Sullivan and Fitzgerald, 1998; Jenkins et al., 2002). The FTIR approach permitted collection of complex biochemical fingerprint-like spectra derived from the total cell composition (Becker et al., 2006), allowing for rapid and reliable typing of Strep. thermophilus starter cultures.

Multivariate analysis of Lactobacillus spp. spectra generated a model that classified the strains according to starters and nonstarters. The model (Figure 3) showed clear separation between L. helveticus starter cultures from the remaining nonstarter strains. However, the nonstarter L. delbrueckii strains formed a separate cluster distinct from both the starters and no-starters. The ICD values between the 3 clusters are shown in Table 5. We observed that L. delbrueckii clustered closer to the L. helveticus starter cultures (ICD 5.1) than to the other nonstarter cultures (ICD 6.7). Lactobacillus delbrueckii strains are used as starter cultures along with $L$. helveticus in the manufacture of Swiss-type cheeses (Khalid and Marth, 1990). Marked differences were observed among the L. delbrueckii strains: the strains were separated in 2 groups with significant interclass separation between strains C2 and F44 and strains E3 and F1 (ICD 15.4), indicating phenotypic differences between these strains. Strains of L. delbrueckii $\mathrm{C} 2$ and F44 showed greater relatedness to the starters than the remaining 2 strains (E3 and F1). Thus, SIMCA analysis provided a model that distinguished the L. delbrueckii strains according to their closeness to the starters (Figure 3).

The SIMCA analysis of the spectral data from Lactobacillus strains also permitted development of a 
A

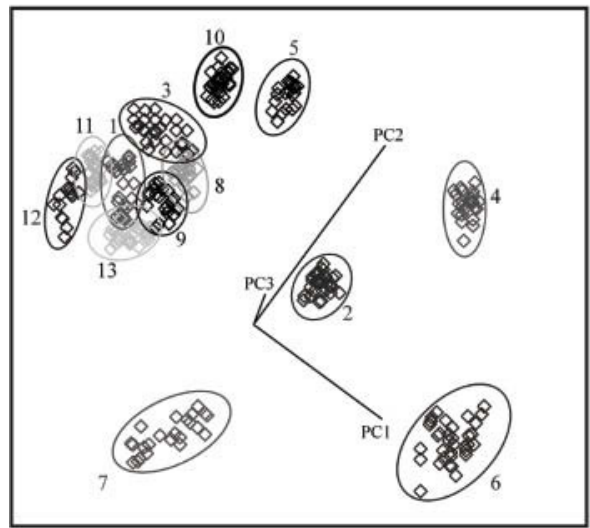

C

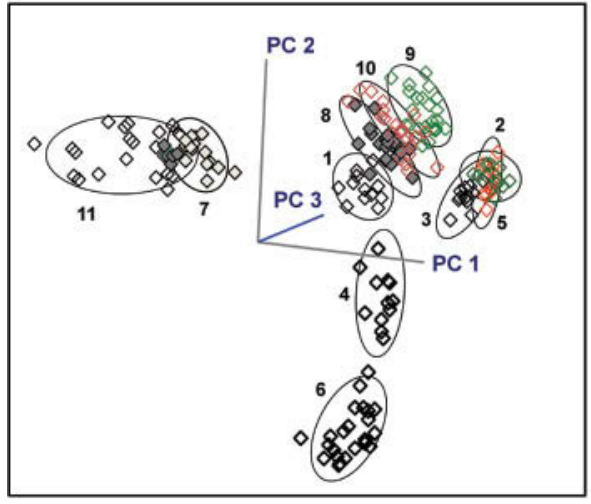

B

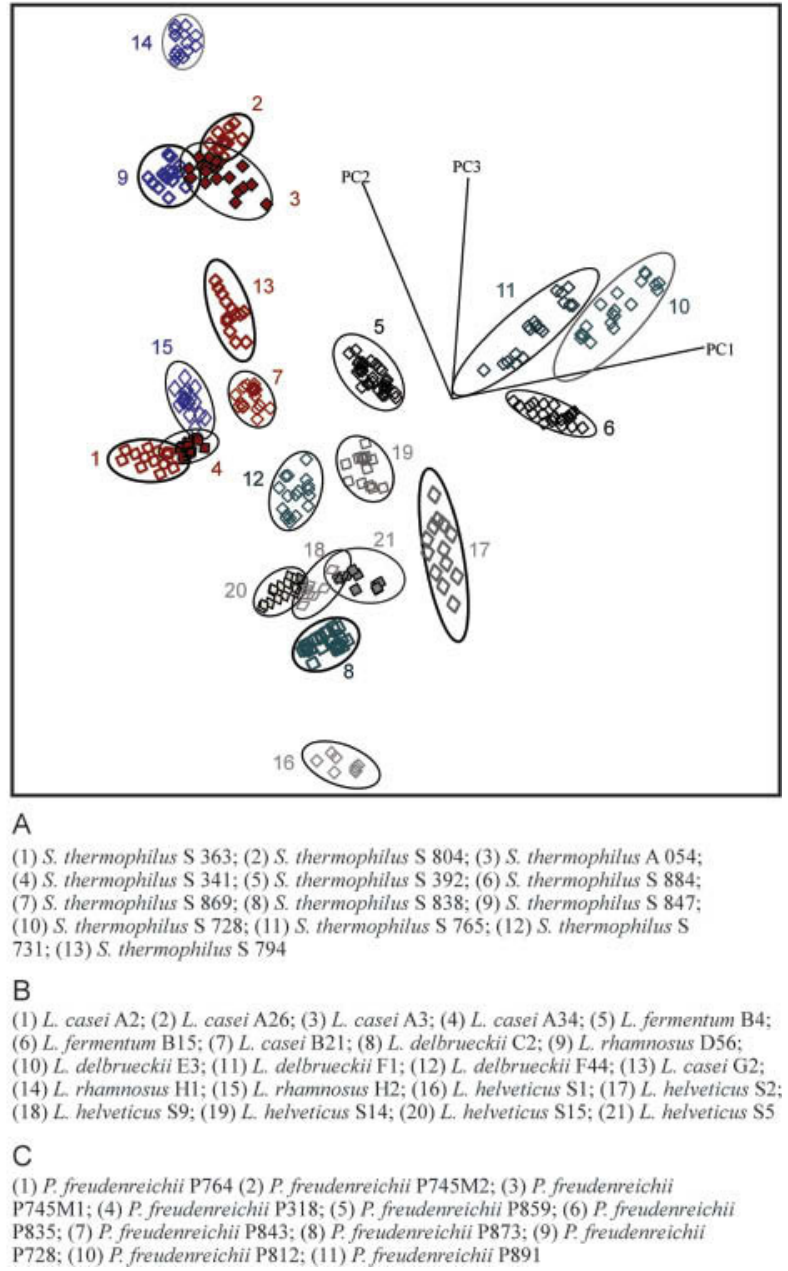

Figure 2. Soft independent modeling of class analogy plot for (A) Streptococcus thermophilus (13) strains, (B) Lactobacillus spp. (21) strains, and (C) Propionibacterium freudenreichii (10) strains. Color version available in the online PDF.

model that classified all strains into individual clusters (Figure 2B). The clustering pattern showed similarities to the SIMCA model that classified the strains according to starters and nonstarters. The 6 starter $L$. helveticus strains and L. delbrueckii $\mathrm{C} 2$ and F44 strains formed close clusters $(8,12$, and 16-21 in Figure 2B). Lactobacillus delbrueckii E3 and F1 strains clustered together (10 and 11 in Figure 2B). These data were also supported by the ICD values (Table 5) provided by multivariate analysis. Interclass difference values $<3$ were observed in closely related lactobacilli strains. Lactobacillus strains are more closely related to each other (55-100\% similarity) than other cheese starter cultures, indicating less heterogeneity within species by PFGE (Jenkins et al., 2002).

The SIMCA analysis of the spectral data from $P$. freudenreichii strains generated a model that classified them into 10 classes (Figure 2C). The model showed good class separation and tight clustering among the 10 strains with between-class distances ranging from 4.2 to 38.5 . The ICD values of this model are indicated in Table 4. Clustering between P. freundenreichii ssp. shermanii strains was similar to that reported by Jenkins et al. (2002) using PFGE. Strains clustered into 3 groups that included P859, P745M1, and P745M2 (group 1), P843 and P891 (group 2), and P764, P812, P873, and P728 (group 3), suggesting similar biochemical characteristics among strains within each group. Jenkins et al. (2002) reported that $P$. freudenreichii strains showed $>30.5 \%$ similarity by PFGE.

The pattern recognition model showed good discrimination in the infrared region from 1,300 to $800 \mathrm{~cm}^{-1}$, and the wavenumbers having the greatest influence on the classification model can be identified in the dis- 
Table 5. Interclass distances between Lactobacillus starters and nonstarter cultures

\begin{tabular}{lccc}
\hline Class $^{1}$ & 1 & 2 & 3 \\
\hline 1 & 0.0 & & \\
2 & 5.1 & 0.0 & 0.0 \\
3 & 6.7 & 5.9 & 0.9 \\
\hline
\end{tabular}

${ }^{1}$ Class $1=$ nonstarter lactic acid bacteria; class $2=$ starter lactic acid bacteria; class $3=$ Lactobacillus delbrueckii.

criminating power plot (Figure 4A). The major bands responsible for the classification models were associated to $\mathrm{C}-\mathrm{O}$, C-C stretching, and $\mathrm{C}-\mathrm{O}-\mathrm{H}$ and $\mathrm{C}-\mathrm{O}-\mathrm{O}$ deformation of the cell components involving cell wall polysaccharide components (Udelhoven et al., 2000; Sahu et al., 2006). Classification of 13 Strep. thermophilus strains showed major discriminating bands at 1,146, $1,065,1,045$, and $991 \mathrm{~cm}^{-1}$ (Figure 4A). Signals at 1,065 and $991 \mathrm{~cm}^{-1}$ are characteristic of carbohydrates, with the former band characterized on a biosurfactant produced by Strep. thermopilus strains (Rodrigues et al., 2006; McCann et al., 2007). The classification of Lactobacillus spp. strains (Figure 4B) showed a major band at $1,150 \mathrm{~cm}^{-1}$ that can be associated with signals from carbohydrate $\mathrm{C}-\mathrm{O}$ stretching vibrations of cell wall carbohydrate components (Nichols et al., 1985; Nau- mann et al., 1995, 1996). Work done by Luginbuhl et al. (2006) reported that the infrared region from 1,300 to $900 \mathrm{~cm}^{-1}$ was the most effective in classifying, at the species level, 102 strains of the Lactobacillus acidophilus group, including L. acidophilus, Lactobacillus amylovorus, Lactobacillus crispatus, Lactobacillus gallinarum, Lactobacillus gasseri, L. helveticus, and Lactobacillus johnsonii. It was reported by Amiel et al. (2001) that the region 1,200 to $900 \mathrm{~cm}^{-1}$, containing signals C-O-C and C-O-P stretching vibration from polysaccharides, were selective for classification of dairy Lactobacillus spp. at the species and strain levels. Figure $4 \mathrm{C}$ shows the discriminating power plot indicating the important discrimination bands for the classification model of $P$. freudenreichii strains. The infrared region from 900 to $1,300 \mathrm{~cm}^{-1}$ resulted in a major discrimination band at $1,010 \mathrm{~cm}^{-1}$ associated with the vibrational mode $(\nu)$ of the $\mathrm{C}=\mathrm{O}$ bond $\left(\nu_{\mathrm{C}-\mathrm{OH}}\right)$ of alcohol $(\mathrm{C} 6-\mathrm{O} 6 \mathrm{H}$; Haxaire et al., 2003). Alcohols display intense $\nu_{\mathrm{C}-\mathrm{O}}$ bands in the 1,000-1,120 $\mathrm{cm}^{-1}$ region (Haxaire et al., 2003). This research is the first attempt to classify Propionibacterium cultures using FTIR spectroscopy. Overall, the SIMCA discrimination power showed that classification of Swiss cheese cultures was attributed to vibrations due to differences in the carbohydrate backbone related to bacterial cell wall phenotype. Strong complex absorp-

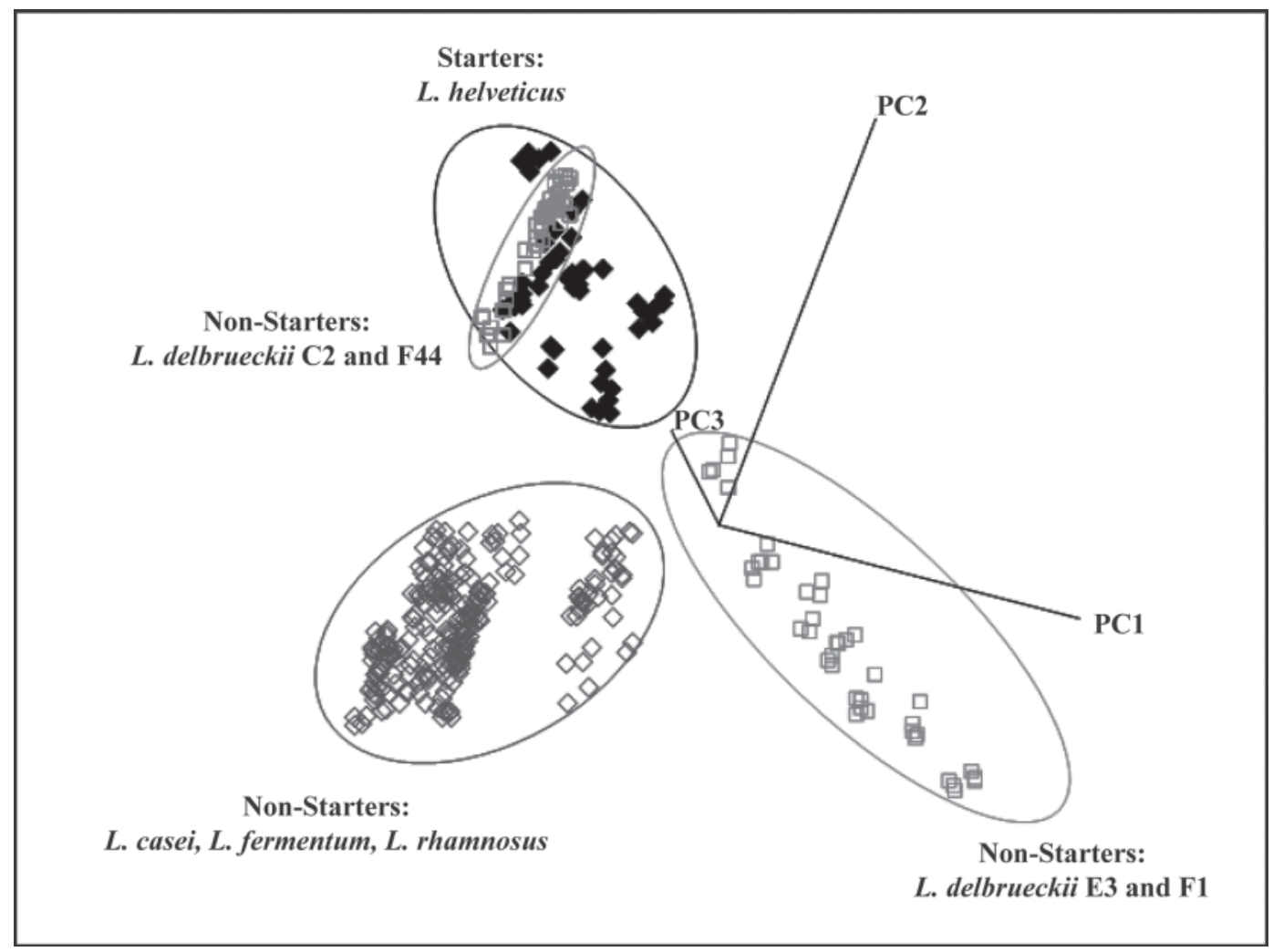

Figure 3. Soft independent modeling of class analogy plot for Lactobacillus starter and nonstarter cultures. 

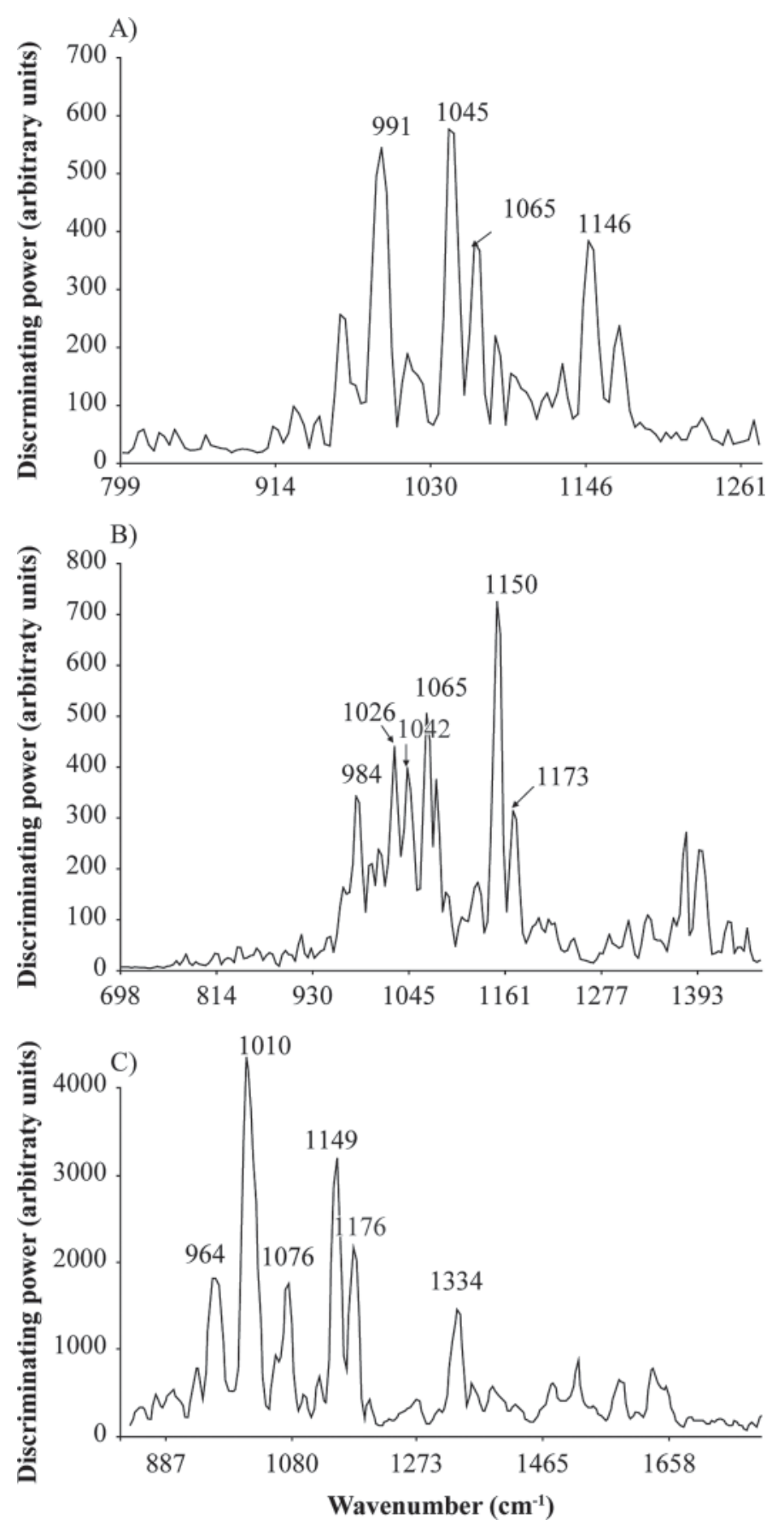

Figure 4. Discriminating power plot for the classification of (A) Streptococcus thermophilus strains, (B) Lactobacillus spp. strains, and (C) Propionibacterium freudenreichii strains. Infrared wavenumbers that were different between classes and the relative extent of the difference are shown.

tion in the 1,100 to $1,000 \mathrm{~cm}^{-1}$ region is ascribed to vibrations of ring structures, and bands between 1,200 and $1,100 \mathrm{~cm}^{-1}$ are assigned to $\nu \mathrm{C}-\mathrm{C}$ and $\nu \mathrm{C}-\mathrm{O}$ weakly coupled to $\delta \mathrm{C}-\mathrm{O}-\mathrm{H}$ modes, including the $\nu \mathrm{C}-\mathrm{O}-\mathrm{C}$ of the glycosidic linkage $\left(\sim 1,124 \mathrm{~cm}^{-1}\right)$.

\section{CONCLUSIONS}

Application of FTIR spectroscopy to classify microorganisms had been successfully implemented by many researchers. This research was aimed at building classification models for several starter and nonstarter cultures used in making Swiss cheese. A rapid and simple method was developed to classify more than 40 Swiss cheese cultures at the strain level using a combination of HGMF and FTIR microspectroscopy. The developed classification model also indicated biochemical similarities between the strains by forming close clusters. The models showed major discrimination in the spectral region from 1,100 to $900 \mathrm{~cm}^{-1}$ associated with stretching vibration signals from polysaccharides in the cell wall. Implementing this technique would enable the Swiss cheese manufacturer to select cheese cultures at the strain level. This information will enable the cheese maker to have a detailed overview of the microbiological status of the starter and nonstarter cultures, which in turn would enable corrective measures to be taken early in the process, limiting production of inferior quality cheese and minimizing defects. Furthermore, this technique could be extended to classify other cheese and dairy cultures.

\section{ACKNOWLEDGMENT}

The authors acknowledge the financial support from The Ohio Agricultural Research and Development Center Seed Program (Wooster, OH) for their financial support. We are also grateful for the support of the Swiss Cheese Consortium (Columbus, $\mathrm{OH}$ ) and the J. T. Parker Chair in Dairy Foods (Columbus, OH).

\section{REFERENCES}

Amiel, C., L. Mariey, C. Denis, P. Patricia, and J. Travert. 2001. FTIR spectroscopy and taxonomic purpose: Contribution to the classification of lactic acid bacteria. Lait 81:249-255.

Becker, K., N. Al Laham, W. Fegeler, R. A. Proctor, G. Peters, and C. von Eiff. 2006. Fourier-transform infrared spectroscopic analysis is a powerful tool for studying the dynamic changes in Staphylococcus aureus small-colony variants. J. Clin. Microbiol. 44:3274-3278.

Beresford, T. P., N. A. Fitzsimons, N. L. Brennan, and T. M. Cogan. 2001. Recent advances in cheese microbiology. Int. Dairy J. 11:259-274.

De Maesschalck, R., A. Candolfi, D. L. Masart, and S. Heuerding. 1999. Decision criteria for soft independent modeling of class analogy applied to near infrared data. Chemom. Intell. Lab. Syst. 47:65-77.

Dunn, W. J., III, and S. Wold. 1995. SIMCA pattern recognition and classification. Pages 179-193 in Chemometric Methods in Molecular Design. H. van der Waterbeemd, ed. VCH Publishers, New York, NY.

Dziuba, B., A. Babuchowski, D. Nalecz, and M. Niklewicz. 2007. Identification of lactic acid bacteria using FTIR spectroscopy and cluster analysis. Int. Dairy J. 17:183-189.

Fox, P. F., T. Guinee, T. Cogan, and P. McSweeney. 2000. Fundamentals of Cheese Science. Aspen Publisher Inc., Gaithersburg, MD. 
Haxaire, K., Y. Marechal, M. Milas, and M. Rinaudo. 2003. Hydration of polysaccharide hyaluronan observed by IR spectrometry. I. Preliminary experiments and band assignments. Biopolymers 72:10-20.

Helm, D., H. Labischinski, and D. Naumann. 1991. Elaboration of a procedure for identification of bacteria using Fourier-transform IR spectral libraries: a stepwise correlation approach. J. Microbiol. Methods 14:127-142.

Holzapfel, W. H., P. Haberer, R. Geisen, J. Bjorkroth, and U. Schillinger. 2001. Taxonomy and important features of probiotic microorganisms in food and nutrition. Am. J. Clin. Nutr. 73:365S$373 \mathrm{~S}$.

Jenkins, J. K., W. J. Harper, and P. D. Courtney. 2002. Genetic diversity in Swiss cheese starter cultures assessed by pulsed field gel electrophoresis and arbitrarily primed PCR. Lett. Appl. Microbiol. 35:423-427.

Ji, T., V. B. Alvarez, and W. J. Harper. 2004. Influence of starter culture ratios and warm room treatment on free fatty acid and amino acid in Swiss cheese. J. Dairy Sci. 87:1986-1992.

Kahala, M., M. Maki, A. Lehtovaara, J.-M. Tapanainen, R. Katiska, M. Juuruskorpi, J. Juhola, and V. Joutsjoki. 2008. Characterization of starter lactic acid bacteria from the Finnish fermented milk product viili. J. Appl. Microbiol. 105:1929-1938.

Kansiz, M., J. R. Gapes, D. McNaughton, B. Lendl, and K. C. Schuster. 2001. Mid infrared spectroscopy coupled to sequential injection analysis for the online monitoring of the acetone-butanol fermentation process. Anal. Chim. Acta 438:175-186.

Khalid, N. M., and E. H. Marth. 1990. Lactobacilli-Their enzymes and role in ripening and spoilage of cheese: A review. J. Dairy Sci. 73:2669-2684.

Kieronczyk, A., S. Skeie, T. Langsrud, and M. Yvon. 2003. Cooperation between Lactococcus lactis and nonstarter lactobacilli in the formation of cheese aroma from amino acids. Appl. Environ. Microbiol. 69:734-739.

Kirschner, C., K. Maquelin, P. Pina, N. A. Ngo Thi, L.-P. ChooSmith, G. D. Sockalingum, C. Sandt, D. Ami, F. Orsini, S. M. Doglia, P. Allouch, M. Mainfait, G. J. Puppels, and D. Naumann. 2001. Classification and identification of enterococci: A comparative phenotypic, genotypic, and vibrational spetroscopic study. J. Clin. Microbiol. 39:1763-1770.

Kyalheim, O. M., and T. V. Karstang. 1992. SIMCA - classification by means of disjoint cross-validated principal components models Pages 209-248 in Multivariate Pattern Recognition in Chemometrics: Illustrated by Case Studies. R. G. Brereton, ed. Elsevier, Amsterdam, the Netherlands.

Lick, S. 2003. Review: Typing systems for lactobacilli. Milchwissenschaft 58:256-260.

Lortala, S., and M. P. Chapot-Chartier. 2005. Role, mechanisms and control of lactic acid bacteria lysis in cheese. Int. Dairy J. 15:857871.

Luginbuhl, W., J. Jimeno, and U. Zehntner. 2006. Identification of seven species of the Lactobacillus acidophilus group by FT-IR spectroscopy. Lebenson. Wiss. Technol. 39:152-158.

Lynch, C. M., P. L. H. McSweeney, P. F. Fox, T. M. Cogan, and F. D. Drinan. 1997. Contribution of starter lactococci and non-starter lactobacilli to proteolysis in Cheddar cheese with a controlled microflora. Lait 77:441-459.

Männig, A., N. A. Baldauf, L. A. Rodriguez-Romo, A. E. Yousef, and L. E. Rodríguez-Saona. 2008. Differentiation of Salmonella enterica serovars and strains in cultures and food using infrared spectroscopic and microspectroscopic techniques combined with soft independent modeling of class analogy pattern recognition analysis. J. Food Prot. 71:2249-2256.

Mannu, L., and A. Paba. 2002. Genetic diversity of lactococci and enterococci isolated from home-made Pecorino Sardoewes' milk cheese. J. Appl. Microbiol. 92:55-62.

McCann, M. C., M. Defernez, B. R. Urbanowicz, J. C. Tewari, T. Langewisch, A. Olek, B. Wells, R. H. Wilson, and N. C. Carpita. 2007. Neural network analyses of infrared spectra for classifying cell wall architectures. Plant Physiol. 143:1314-1326.
Mossoba, M. M., S. F. Al-Khaldib, A. Jacobson, L. I. Segarra-Crowe, and F. S. Fry. 2003. Application of a disposable transparent filtration membrane to the infrared spectroscopic discrimination among bacterial species. J. Microbiol. Methods 55:311-314.

Naumann, D., D. Helm, and H. Labischinski. 1991. Elaboration of a procedure for identification of bacteria using Fourier-transform IR spectral libraries: A stepwise correlation approach . Nature 351:81-82.

Naumann, D., S. Keller, D. Helm, C. Schultz, and B. Schrader. 1995 FT-IR spectroscopy and FT-Raman spectroscopy are powerful analysis tools for the non-invasive characterization of intact microbial cells. J. Mol. Struct. 347:399-406.

Naumann, D., P. C. Schultz, and D. Helm. 1996. What can infrared spectroscopy tells us about the structure and composition of intact bacteria cells? Pages 279-310 in Infrared Spectroscopy of Biomolecules. H. H. Mantsh and D. Chapman, ed. Wiley-Liss, New York, NY.

Ngo-Thi, N. A., C. Kirschner, and D. Naumann. 2003. Characterization and identification of microorganisms by FT-IR microspectrometry. J. Mol. Struct. 661:371-380.

Nichols, P. D., J. M. Henson, J. B. Guckert, D. E. Nivens, and D. C. White. 1985. Fourier transform-infrared spectroscopic methods for microbial ecology: Analysis of bacteria, bacteria-polymer mixtures and biofilms. J. Microbiol. Methods 4:79-94.

O'Sullivan, T. F., and G. F. Fitzgerald. 1998. Comparison of Streptococcus thermophilus strains by pulse field gel electrophoresis of genomic DNA. FEMS Microbiol. Lett. 168:213-219.

Oust, A., T. Møretrø, C. Kirschner, J. A. Narvhus, and A. Kohler. 2004. FT-IR spectroscopy for identification of closely related lactobacilli. J. Microbiol. Methods 59:149-162.

Rodrigues, L. R., J. A. Teixeira, H. C. van der Mei, and R. Oliveira. 2006. Isolation and partial characterization of a biosurfactant produced by Streptococcus thermophilus A. Colloids Surf. B Biointerfaces 53:105-112.

Sahu, R. K., S. Mordechai, S. Pesakhov, R. Dagan, and N. Porat. 2006. Use of FTIR spectroscopy to distinguish between capsular types and capsular quantities in Streptococcus pneumoniae. Biopolymers 83:434-442.

Steffen, C., E. Flueckiger, J. Bosset, and M. Ruegg. 1987. Swiss-type varieties. Pages 93-120 in Cheese: Chemistry, Physics and Microbiology. Vol. 2. P. F. Fox, ed. Elsevier Applied Science Publishers Ltd., New York, NY.

Tannock, G. W. 2001. Molecular assessment of intestinal microflora Am. J. Clin. Nutr. 73:410S-414S.

Tanskanen, E. I., D. L. Tulloch, A. J. Hillier, and B. E. Davidson. 1990. Pulsed-field gel electrophoresis of SmaI digests of lactococcal genomic DNA, a novel method of strain identification. Appl. Environ. Microbiol. 56:3105-3111.

Tilsala-Timisjarvi, A., and T. Alatossava. 1997. Development of oligonucleotide primers from the 16S-23S rRNA intergenic sequences for identifying different dairy and probiotic lactic acid bacteria by PCR. Int. J. Food Microbiol. 35:49-56.

Udelhoven, T., D. Naumann, and J. Schmitt. 2000. Development of a hierarchical classification system with artificial neural networks and FT-IR spectra for the identification of bacteria. Appl. Spectrosc. 54:1471-1479.

USDA. 2001. United States Standards for Swiss Cheese, Emmentaler Cheese. Accessed October 2010. http://www.ams.usda.gov/ AMSv1.0/getfile?dDocName=STELDEV3004468.

Ward, L. J. H., H. A. Heap, and W. J. Kelly. 2004. Characterization of closely related lactococcal starter strains which show differing patterns of bacteriophage sensitivity. J. Appl. Microbiol. 96:144-148.

Weinrichter, B., H. Sollberger, W. Ginzinger, D. Jaros, and H. Rohm., 2004. Adjunct starter properties affect characteristic features of Swiss-type cheeses. Food/Nahrung 48:73-79.

Wenning, M., H. Seiler, and S. Scherer. 2002. Fourier-transform infrared microspectroscopy, a novel and rapid tool for identification of yeasts. Appl. Environ. Microbiol. 68:4717-4721. 